\title{
Влияние замещения висмута празеодимом и лантаном на термоэлектрические свойства оксиселенидов BiCuSeO
}

\author{
(C) А.П. Новицкий ${ }^{1,2}$, И.А. Сергиенко ${ }^{1}$, С.В. Новиков ${ }^{2}$, К.В. Кусков ${ }^{1}$, Д.В. Лейбо ${ }^{1}$, \\ Д.С. Панкратова ${ }^{1}$, А.Т. Бурков ${ }^{2}$, В.В. Ховайло ${ }^{1}$ \\ ${ }^{1}$ Национальный исследовательский технологический университет „МИСиС“, \\ 119049 Москва, Россия \\ ${ }^{2}$ Физико-технический институт им. А.Ф. Иофффе Российской академии наук, \\ 194021 Санкт-Петербург, Россия \\ E-mail: novitskiy@misis.ru
}

(Получена 13 июня 2018 г. Принята к печати 18 июня 2018 г.)

\begin{abstract}
Представлены результаты исследования термоэлектрических свойств объемных образцов оксиселенидов $\mathrm{Bi}_{1-x} \operatorname{Pr}_{x} \mathrm{CuSeO}(x=0,0.04,0.08)$ и $\mathrm{Bi}_{0.96} \mathrm{La}_{0.04} \mathrm{CuSeO} p$-типа проводимости, полученных методом твердофазного синтеза. Измерены температурные зависимости термоэдс, электросопротивления и теплопроводности от комнатной температуры до $800 \mathrm{~K}$. Во всем интервале температур наблюдается снижение удельного электросопротивления и термоэдс с увеличением концентрации замещающего элемента, в то время как теплопроводность при замещении висмута редкоземельными элементами практически не изменяется. Несмотря на то что номинальная валентность $\mathrm{Bi}$, La и $\operatorname{Pr}$ одинакова, замещение висмута ионами редкоземельных элементов приводит к увеличению концентрации носителей заряда, что может быть вызвано разницей электронных конфигураций ионов, и, как следствие, смещением уровня Ферми в валентную зону.
\end{abstract}

DOI: $10.21883 /$ FTP.2019.02.47103.8932

\section{1. Введение}

В последнее время медные оксиселениды привлекают значительное внимание научного сообщества благодаря их низкой теплопроводности и относительно высокому значению термоэдс. Более того, исследования оксиселенидов $\mathrm{BiCuSeO}$ показали, что эти материалы обладают высокой термоэлектрической эффективностью при температурах до $923 \mathrm{~K}$ [1-3]. Медные оксиселениды $\mathrm{BiCuSeO}$ относятся к семейству оксихалькогенидов $\mathrm{LnCuChO}$ (где Ln - ион редкоземельного элемента или висмута, Ch - ион группы халькогенидов или пниктогенов), кристаллическая решетка которых соответствует структурному типу $\mathrm{ZrSiCuAs}$ (пространственная группа $P 4 / n m m)$ и состоит из расположенных вдоль оси $c$ тетрагональной кристаллической ячейки слоев $\left(\mathrm{Cu}_{2} \mathrm{Ch}_{2}\right)^{2-}$ и $\left(\mathrm{Ln}_{2} \mathrm{O}_{2}\right)^{2+}$ (рис. 1) [4]. Основное ограничение для применения данных материалов в качестве термоэлектрических - их высокое удельное электросопротивление $\sim 50$ мОм $\cdot$ см. В ряде работ было показано, что увеличение электропроводности может быть достигнуто путем увеличения концентрации носителей заряда за счет замещения ионов $\mathrm{Bi}^{3+}$ ионами $\mathrm{Sr}^{2+}, \mathrm{Ba}^{2+}, \mathrm{Pb}^{2+}$, $\mathrm{Ca}^{2+}$ или $\mathrm{Na}^{+}[2,5-8]$. Однако подвижность носителей заряда значительно снижается при таком замещении, тем самым ограничивая увеличение термоэлектрической эффективности $z T$. Данное снижение может быть обусловлено различием электронных конфигураций $\mathrm{Bi}^{3+}$ и $\mathrm{A}^{2+}$, где $\mathrm{A}$ - ион щелочно-земельного металла. Более того, предел растворимости дивалентных и моновалентных ионов не превышает 10 и 6\% соответственно [9]. Таким образом, целесообразно подбирать легирующий элемент с близкой к $\mathrm{Bi}^{3+}$ электронной структурой и высоким пределом растворимости. Наиболее перспек- тивными кандидатами на эту роль являются ионы редкоземельных элементов $[4,10]$.

В данной работе было проведено исследование влияния замещения ионов висмута ионами празео-

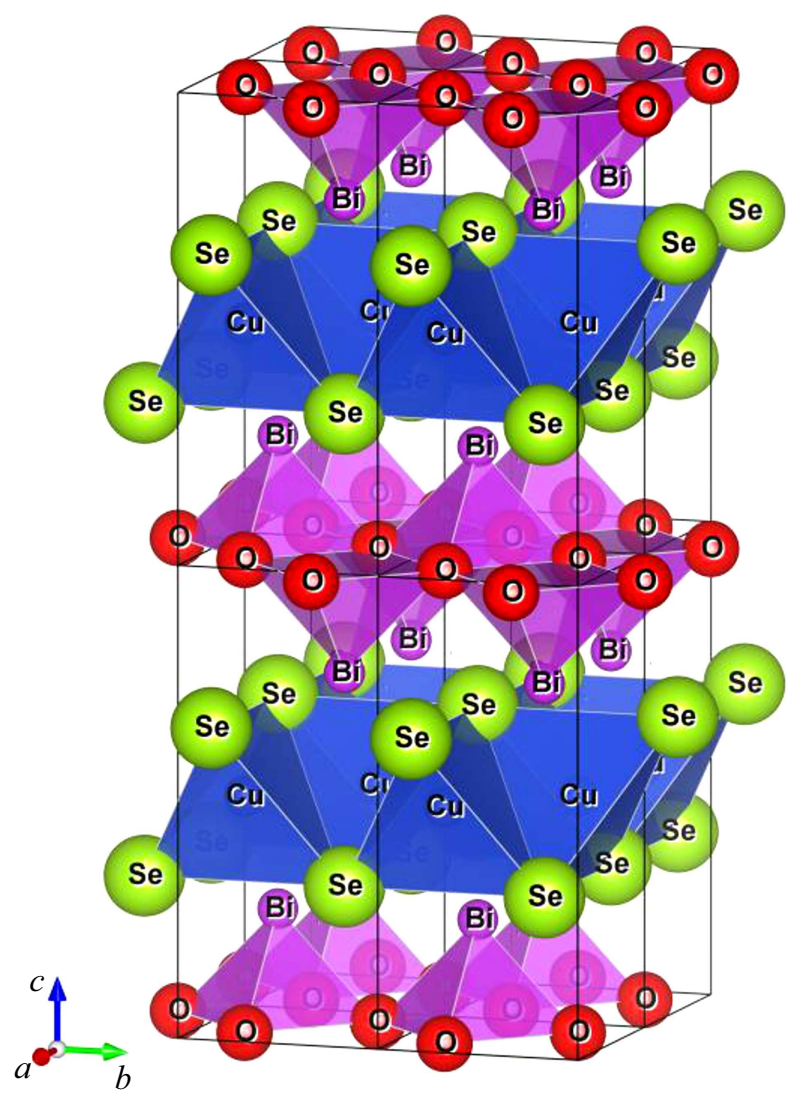

Рис. 1. Кристаллическая структура $\mathrm{BiCuSeO}$. 
дима на термоэлектрические свойства $\mathrm{Bi}_{1-x} \mathrm{Pr}_{x} \mathrm{CuSeO}$ $(x=0,0.04,0.08)$ в интервале температур $300-800 \mathrm{~K}$. В ряде работ было показано, что легирование немагнитных полупроводниковых соединений элементами с ненулевым магнитным моментом может приводить к дополнительному повышению коэффициента термоэдс за счет дополнительного увеличения эффективной массы носителей заряда или спиновой энтропии вносимой магнитными ионами $[11,12]$. С целью установления влияния магнитного момента празеодима на электрофизические свойства был также синтезирован и исследован образец $\mathrm{Bi}_{0.96} \mathrm{La}_{0.04} \mathrm{CuSeO}$, учитывая, что масса и электронная структура лантана и празеодима близки, но магнитный момент лантана равен нулю.

\section{2. Образцы и методы исследования}

Образцы $\mathrm{Bi}_{1-x} \operatorname{Pr}_{x} \mathrm{CuSeO} \quad(x=0,0.04,0.08) \quad$ и $\mathrm{Bi}_{0.96} \mathrm{La}_{0.04} \mathrm{CuSeO}$ были синтезированы методом двухступенчатого твердофазного синтеза с последующим искровым плазменным спеканием. В качестве исходных реактивов использовались мелкодисперсные порошки оксидов $\mathrm{Bi}_{2} \mathrm{O}_{3}, \mathrm{La}_{2} \mathrm{O}_{3}, \mathrm{Pr}_{2} \mathrm{O}_{3}$ и чистых элементов $\mathrm{Bi}, \mathrm{Se}$, и $\mathrm{Cu}$. Стехиометрическая смесь порошков подвергалась холодному прессованию в образец цилиндрической формы. Отжиг образцов проводился в запаянной кварцевой ампуле ( 10-3 Торр) в течение 8 ч при температуре $573 \mathrm{~K}$. Полученные образцы размалывались в порошок с помощью планетарной микромельницы, подвергались повторным холодному прессованию и отжигу в запаянной кварцевой ампуле в течение 12 ч при температуре $973 \mathrm{~K}$. Полученные однофазные образцы $\mathrm{Bi}_{1-x} \operatorname{Pr}_{x} \mathrm{CuSeO}$ $(x=0,0.04,0.08)$ и $\mathrm{Bi}_{0.96} \mathrm{La}_{0.04} \mathrm{CuSeO}$ были размолоты в порошок с использованием планетарной микромельницы Fritsch Pulverisette 7 premium line. Помол проводился в атмосфере аргона в течение 8 ч со скоростью 300 об/мин, параметры выбирались в соответствии с литературными данными [13]. С целью получения объемных образцов проводилось искровое плазменное спекание полученных порошков при температуре $973 \mathrm{~K}$ в течение 5 мин с использованием одноосного давления 50 МПа. Полученные в результате образцы в форме цилиндра с диаметром и высотой $12.7 \times 10$ мм подвергались отжигу в кварцевой ампуле в атмосфере аргона при температуре $973 \mathrm{~K}$ в течение 6 ч. Фазовый состав определялся методом рентгеновской дифракции на дифрактометре Дифрей 401 с использованием $\mathrm{Cr} K_{\alpha}$ $(\lambda=2.2911 \AA)$ излучения при комнатной температуре. Температурная зависимость удельного электросопротивления измерялась четырехзондовым методом, измерения коэффициента термоэдс проводились с использованием дифференциального метода [14]. Ошибка в определении термоэдс и электросопротивления не превышает 5 и 2\% соответственно. Температурная зависимость температуропроводности измерялась методом лазерной вспышки, с целью минимизировать ошибки, вносимые за счет отражения или частичной прозрачности образцов, они бы- ли покрыты тонким слоем графита. Полученные данные анализировались по модели Кована с учетом коррекции импульса. Теплопроводность определялась из измерений температуропроводности по формуле $\kappa=\chi \cdot C_{p} \cdot d$, где $\chi-$ температуропроводность $\left(\mathrm{MM}^{2} \cdot \mathrm{c}^{-1}\right), C_{p}-$ удельная теплоемкость (Дж $\left.\cdot \Gamma^{-1} \cdot \mathrm{K}^{-1}\right), d-$ объемная плотность $\left(\Gamma \cdot \mathrm{cm}^{-3}\right)$. Теплоемкость рассчитывалась по теории Дебая, плотность определялась методом гидростатического взвешивания. Для нелегированного образца $\mathrm{BiCuSeO}$ теоретически рассчитанные значения теплоемкости хорошо совпадают с экспериментальными данными [15,16], при температурах выше комнатной теплоемкость достигает значения, соответствующего закону Дюлонга-Пти, и равна 0.271 Дж/(г $\cdot$ К). Ошибка в определении теплопроводности составляет 8\%. Bce измерения проводились в интервале температур 300-800 K в перпендикулярном направлении относительно оси прессования во время процесса искрового плазменного спекания. Концентрация и подвижность носителей заряда определялись из измерений эффекта Холла по формулам $p=1 / e R_{\mathrm{H}}$ и $\mu=\sigma R_{\mathrm{H}}$, где $p$ - концентрация носителей заряда $\left(\mathrm{cm}^{-3}\right), R_{\mathrm{H}}-$ константа Холла $\left(\mathrm{cm}^{3} \cdot \mathrm{Kл}^{-1}\right), \mu$ - подвижность носителей заряда $\left(\mathrm{cm}^{2} \cdot \mathrm{B}^{-1} \cdot \mathrm{c}^{-1}\right), \sigma$ - электропроводность $\left(\mathrm{OM}^{-1} \cdot \mathrm{cm}^{-1}\right)$. Суммарно, погрешность в определении термоэлектрической эффективности $z T$ не более $15 \%$.

\section{3. Результаты исследований}

На рис. 2 представлены дифрактограммы поликристаллических образцов $\mathrm{Bi}_{1-x} \operatorname{Pr}_{x} \mathrm{CuSeO}(x=0,0.04$, $0.08)$ и $\mathrm{Bi}_{0.96} \mathrm{La}_{0.04} \mathrm{CuSeO}$ после искрового плазменного

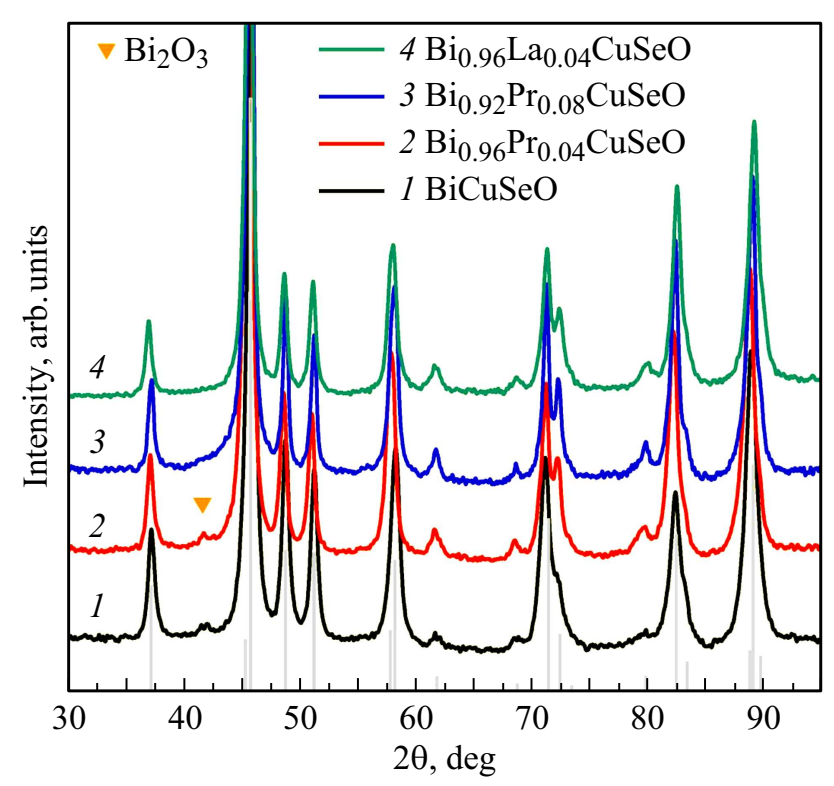

Рис. 2. Дифрактограммы образцов $\mathrm{BiCuSeO}$ (1), $\mathrm{Bi}_{0.96} \operatorname{Pr}_{0.04} \mathrm{CuSeO} \quad(2), \quad \mathrm{Bi}_{0.92} \mathrm{Pr}_{0.08} \mathrm{CuSeO} \quad$ (3) и $\mathrm{Bi}_{0.96} \mathrm{La}_{0.04} \mathrm{CuSeO}(4)$. 
Таблица 1. Решеточная и электронная составляющие теплопроводности при температурах 298 и $800 \mathrm{~K}$ для исследуемых образцов

\begin{tabular}{c|c|c|c|c|c|c|c|c}
\hline \multirow{2}{*}{ Замещение } & \multicolumn{4}{|c|}{$298 \mathrm{~K}$} & \multicolumn{4}{c}{$800 \mathrm{~K}$} \\
\cline { 2 - 9 } & 0 & $0.04 \operatorname{Pr}$ & $0.04 \mathrm{La}$ & $0.08 \operatorname{Pr}$ & 0 & $0.04 \operatorname{Pr}$ & $0.04 \mathrm{La}$ & $0.08 \operatorname{Pr}$ \\
\hline$\kappa_{l}, \mathrm{BT} /(\mathrm{M} \cdot \mathrm{K})$ & 1.14 & 0.95 & 0.95 & 0.98 & 0.65 & 0.65 & 0.62 & 0.65 \\
$\kappa_{e}, \mathrm{BT} /(\mathrm{M} \cdot \mathrm{K})$ & 0.02 & 0.03 & 0.03 & 0.05 & 0.03 & 0.04 & 0.04 & 0.06
\end{tabular}

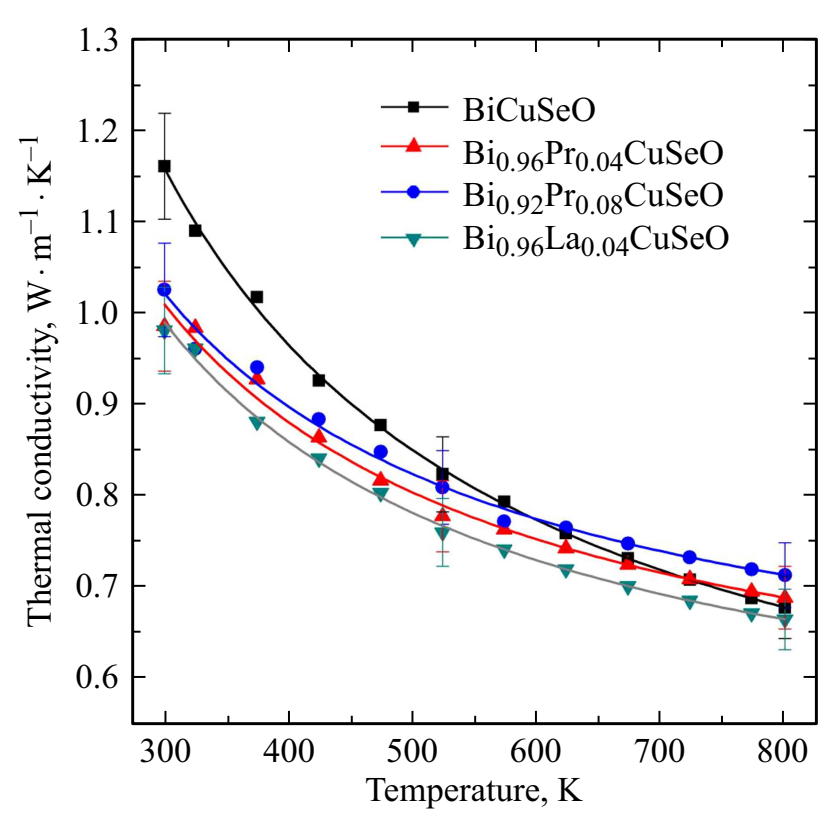

Рис. 3. Температурная зависимость теплопроводности образцов $\mathrm{Bi}_{1-x} \operatorname{Pr}_{x} \mathrm{CuSeO}(x=0,0.04,0.08)$ и $\mathrm{Bi}_{0.96} \mathrm{La}_{0.04} \mathrm{CuSeO}$.

спекания и отжига. Положение основных пиков соответствует фазе $\mathrm{BiCuSeO}$ (PDF 45-0296), однако в образцах $\mathrm{BiCuSeO}$ и $\mathrm{Bi}_{0.96} \operatorname{Pr}_{0.04} \mathrm{CuSeO}$ обнаружено незначительное количество $\mathrm{Bi}_{2} \mathrm{O}_{3}$.

Температурные зависимости теплопроводности для всех образцов представлены на рис. 3. Для соединений на основе $\mathrm{BiCuSeO}$ вклад фононной (решеточной) теплопроводности - основной, о чем можно судить по виду температурной зависимости теплопроводности $\kappa \propto T^{-1}$, что также подтверждено расчетами электронной составляющей теплопроводности $\kappa_{e}$ по закону Видемана-Франца. В соответствии с моделью теплопроводности неметаллических соединений $[17,18]$ решеточная теплопроводность пропорциональна атомной массе по формуле

$$
\kappa_{l}=A \frac{\bar{M} \theta_{D}^{3} V^{1 / 3}}{\gamma^{2} n^{2 / 3} T},
$$

где $n$ - количество атомов в элементарной ячейке, $V$ - объем в расчете на один атом, $\theta_{D}$ - температура Дебая, $\bar{M}$ - средняя атомная масса, $\gamma$ - параметр Грюнайзена и $A-$ константа. Атомные массы висмута, лантана и празеодима равны 208.980, 138.905, 140.908 г/моль соответственно. В свою очередь ионные радиусы ионов $\mathrm{Bi}^{3+}, \mathrm{La}^{3+}$ и $\operatorname{Pr}^{3+}$ близки: 1.03, 1.03 и $0.99 \AA$ соответственно. Учитывая разность атомных масс и малую степень замещения, а также фактически одинаковые ионные радиусы висмута, лантана и празеодима, замещение ионов висмута редкоземельными ионами должно приводить к незначительному снижению решеточной теплопроводности легированных образцов. В табл. 1 показаны значения электронной и решеточной составляющих теплопроводности при температурах 298 и $800 \mathrm{~K}$ для всех образцов. Из табл. 1 видно, что теплопроводность легированных образцов незначительно ниже по сравнению с нелегированным, однако при высоких температурах решеточная теплопроводность для всех образцов фактически одинакова и равна $0.65 \mathrm{BT} /(\mathrm{M} \cdot \mathrm{K})$ при температуре $800 \mathrm{~K}$.

Результаты измерений температурных зависимостей термоэдс и удельного электросопротивления показаны на рис. 4,5. Для всех образцов при температурах до $650 \mathrm{~K}$ наблюдается металлический характер температурной зависимости электросопротивления, однако при увеличении температуры характер электросопротивления изменяется на полупроводниковый. При этом пик

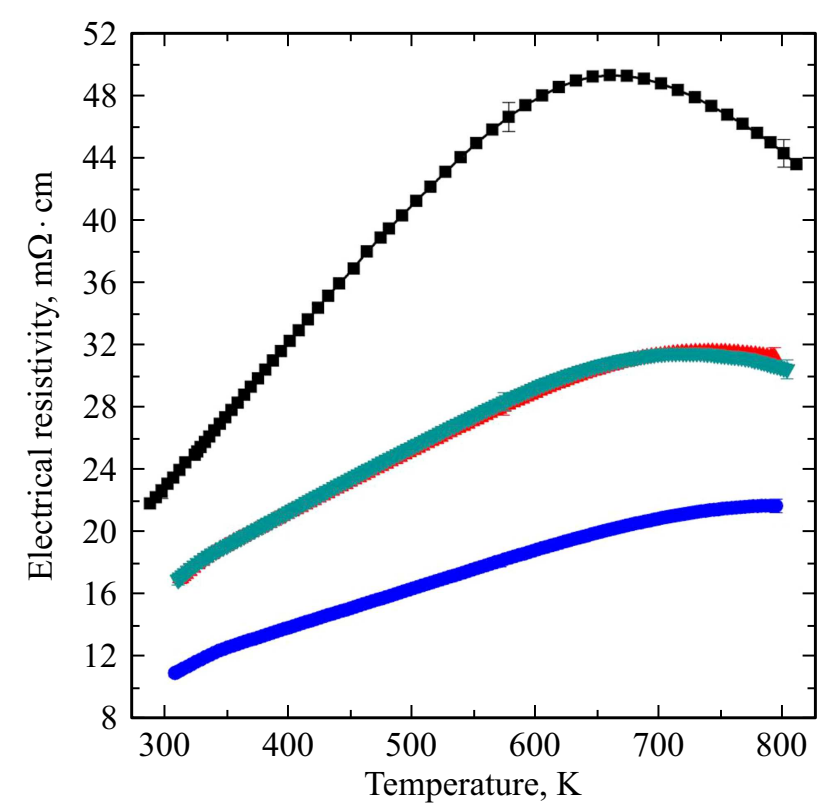

Рис. 4. Зависимость удельного электросопротивления от температуры для $\mathrm{Bi}_{1-x} \operatorname{Pr}_{x} \mathrm{CuSeO} \quad(x=0,0.04,0.08)$ и $\mathrm{Bi}_{0.96} \mathrm{La}_{0.04} \mathrm{CuSeO}$, обозначения для образцов разных составов такие же как на рис. 3. 


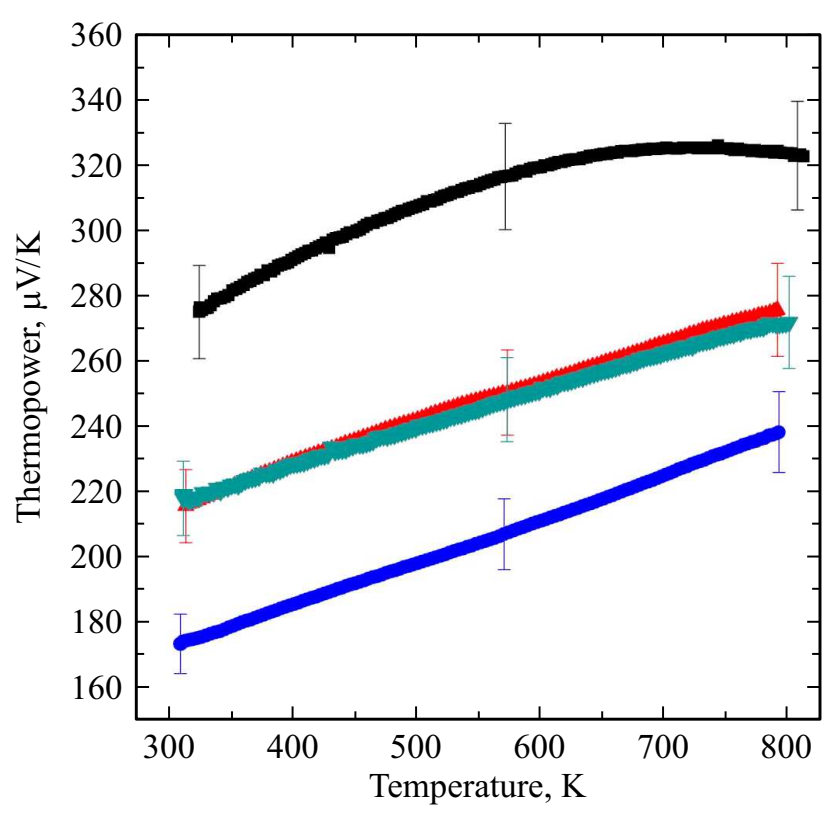

Рис. 5. Температурная зависимость коэффициента термоэдс для $\mathrm{Bi}_{1-x} \operatorname{Pr}_{x} \mathrm{CuSeO}(x=0,0.04,0.08)$ и $\mathrm{Bi}_{0.96} \mathrm{La}_{0.04} \mathrm{CuSeO}$, обозначения для образцов разных составов такие же как на рис. 3.

электросопротивления смещается в сторону более высоких температур при замещении ионов висмута. Так, для $\mathrm{Bi}_{0.96} \mathrm{Pr}_{0.04} \mathrm{CuSeO}$ и $\mathrm{Bi}_{0.96} \mathrm{La}_{0.04} \mathrm{CuSeO}$ слабое падение удельного электросопротивления начинается при температуре около $700 \mathrm{~K}$, для $\mathrm{Bi}_{0.92} \mathrm{Pr}_{0.08} \mathrm{CuSeO}$ изменения характера электросопротивления не наблюдается. Замещение ионов $\mathrm{Bi}^{3+}$ на ионы $\mathrm{Pr}^{3+}$ и $\mathrm{La}^{3+}$ приводит к снижению значений удельного электросопротивления и термоэдс во всем интервале температур. Стоит отметить, что результаты измерений для образцов $\mathrm{Bi}_{0.96} \operatorname{Pr}_{0.04} \mathrm{CuSeO}$ и $\mathrm{Bi}_{0.96} \mathrm{La}_{0.04} \mathrm{CuSeO}$ идентичны, по этим данным не представляется возможным выделить эффект влияния магнитного момента празеодима на электрофизические свойства $\mathrm{BiCuSeO}$. Характер наблюдаемых изменений на графиках термоэдс и электросопротивления (рис. 4,5) является признаком увеличения концентрации носителей заряда. Вторым фактором, подтверждающим данное

Таблица 2. Концентрация и подвижность носителей заряда, электропроводность при температуре $298 \mathrm{~K}$ для исследуемых образцов

\begin{tabular}{c|c|c|c}
\hline $\begin{array}{c}\text { Химический } \\
\text { состав }\end{array}$ & $\begin{array}{c}p \cdot 10^{18} \\
\mathrm{~cm}^{-3}\end{array}$ & $\begin{array}{c}\sigma, \\
1 /(\mathrm{OM} \cdot \mathrm{cm})\end{array}$ & $\begin{array}{c}\mu, \\
\mathrm{cm}^{2} /(\mathrm{B} \cdot \mathrm{c})\end{array}$ \\
\hline $\mathrm{BiCuSeO}$ & 1.26 & 44.38 & 219.61 \\
$\mathrm{Bi}_{0.96} \mathrm{La}_{0.04} \mathrm{CuSeO}$ & 12.43 & 62.63 & 31.46 \\
$\mathrm{Bi}_{0.96} \mathrm{Pr}_{0.04} \mathrm{CuSeO}$ & 13.82 & 61.95 & 27.98 \\
$\mathrm{Bi}_{0.92} \mathrm{Pr}_{0.08} \mathrm{CuSeO}$ & 17.46 & 95.36 & 34.08 \\
$\mathrm{Bi}_{0.95} \mathrm{Ba}_{0.05} \mathrm{CuSeO}[2]$ & $\sim 98$ & $\sim 250$ & $\sim 7$ \\
$\mathrm{Bi}_{0.98} \mathrm{Na}_{0.02} \mathrm{CuSeO}[8]$ & $\sim 92$ & $\sim 125$ & $\sim 9$
\end{tabular}

предположение, может служить смещение максимумов на кривых электросопротивления и термоэдс в сторону высоких температур. Определенные по измерениям эффекта Холла значения концентрации и подвижности носителей заряда и электропроводности при комнатной температуре для всех образцов показаны в табл. 2. Для сравнения представлены данные для образцов $\mathrm{Bi}_{0.95} \mathrm{Ba}_{0.05} \mathrm{CuSeO}[2]$ и $\mathrm{Bi}_{0.98} \mathrm{Na}_{0.02} \mathrm{CuSeO}$ [8]. По данным табл. 2 можно сделать вывод, что замещение редкоземельными ионами приводит к увеличению концентрации носителей заряда на порядок по сравнению с нелегированным $\mathrm{BiCuSeO}$. Однако увеличение концентрации носителей заряда при замещении ионов висмута ионами щелочных и щелочно-земельных металлов гораздо выше, что приводит к большим значениям электропроводности. В случае замещения ионов висмута ионами редкоземельных элементов увеличение концентрации может быть вызвано разницей электронных конфигураций ионов, несмотря на то что номинальная валентность одинакова. Разные электронные конфигурации ионов могут являться причиной смещения уровня Ферми в валентную зону, тем самым приводя к повышению концентрации носителей заряда.

\section{4. Заключение}

Исследованы температурные зависимости термоэдс, сопротивления и теплопроводности объемных образцов оксиселенидов $\mathrm{Bi}_{1-x} \operatorname{Pr}_{x} \mathrm{CuSeO}(x=0,0.04,0.08)$ и $\mathrm{Bi}_{0.96} \mathrm{La}_{0.04} \mathrm{CuSeO}$-типа проводимости в интервале температур 300-800 K. Замещение ионов $\mathrm{Bi}^{3+}$ на $\mathrm{Pr}^{3+}$ или $\mathrm{La}^{3+}$ приводит к уменьшению удельного электросопротивления и термоэдс, что в свою очередь является результатом увеличения концентрации носителей заряда. На основании представленных данных не удается выявить вклад магнитного момента празеодима в электрофизические свойства $\mathrm{BiCuSeO}$. На высокотемпературных значениях теплопроводности замещение ионов висмута редкоземельными элементами фактически не сказывается.

Исследование выполнено при финансовой поддержке РФФИ в рамках научного проекта № 17-32-50165 ,мол_нр“.

\section{Список литературы}

[1] X. Zhang, C. Chang, Y. Zhou, L.-D. Zhao. Materials (Basel), 10, 198 (2017).

[2] J. Li, J. Sui, Y. Pei, C. Barreteau, D. Berardan, N. Dragoe, W. Cai, J. He, L.-D. Zhao. Energy Environ. Sci., 5, 8543 (2012).

[3] Y.L. Pei, H. Wu, D. Wu, F. Zheng, J. He. J. Am. Chem. Soc., 136, 13902 (2014).

[4] R. Pöttgen, D. Johrendt. Zeitschrift Fur Naturforsch.-Sect. B J. Chem. Sci., 63, 1135 (2008). 
[5] L.D. Zhao, D. Berardan, Y.L. Pei, C. Byl, L. Pinsard-Gaudart, N. Dragoe. Appl. Phys. Lett., 97, 092118 (2010).

[6] S.D.N. Luu, P. Vaqueiro. J. Mater. Chem. A, 1, 12270 (2013).

[7] Y.-L. Pei, J. He, J.-F. Li, F. Li, Q. Liu, W. Pan, C. Barreteau, D. Berardan, N. Dragoe, L.-D. Zhao. NPG Asia Mater., 5, e47 (2013).

[8] J. Li, J. Sui, Y. Pei, X. Meng, D. Berardan, N. Dragoe, W. Cai, L.-D. Zhao. J. Mater. Chem. A, 2, 4903 (2014).

[9] R. Liu, X. Tan, Y. Liu, G. Ren, J. Lan, Z. Zhou, C. Nan, Y. Lin. Rare Met., 37, 259 (2018).

[10] Y. Liu, J. Ding, B. Xu, J. Lan, Y. Zheng, B. Zhan, B. Zhang, Y. Lin, C. Nan. Appl. Phys. Lett., 106, 233903 (2015).

[11] Q. Wen, C. Chang, L. Pan, X. Li, T. Yang, H. Guo, Z.-H. Wang, J. Zhang, F. Xu, Z.-D. Zhang, G. Tang. J. Mater. Chem. A, 5, 13392 (2017).

[12] F. Ahmed, N. Tsujii, T. Mori. J. Mater. Chem. A, 5, 7545 (2017).

[13] F. Li, J.-F. Li, L.-D. Zhao, K. Xiang, Y. Liu, B.-P. Zhang, Y.-H. Lin, C.-W. Nan, H.-M. Zhu. Energy Environ. Sci., 5, 7188 (2012).

[14] A.T. Burkov, A. Heinrich, P.P. Konstantinov, T. Nakama, K. Yagasaki. Meas. Sci. Technol., 12, 264 (2001).

[15] D. Berardan, J. Li, E. Amzallag, S. Mitra, J.H. Sui, W. Cai, N. Dragoe. Materials (Basel), 8, 1043 (2015).

[16] C. Barreteau, D. Beardan, E. Amzallag, L. Zhao, N. Dragoe. Chem. Mater., 24, 3168 (2012).

[17] D.T. Morelli, G.A. Slack. High Therm. Conduct. Mater. (Springer, 2006) p. 37.

[18] G.A. Slack. Solid State Phys.: Adv. Res. Appl., 34, 1 (1979).

Редактор Г.А. Оганесян

\section{Influence of $\mathrm{Bi}$ substitution by $\mathrm{Pr}$ and $\mathrm{La}$ on thermoelectric properties of $\mathrm{BiCuSeO}$ oxyselenides}

A.P. Novitskii ${ }^{1,2}$, I.A. Sergienko ${ }^{1}$, S.V. Novikov ${ }^{2}$, K.V. Kuskov ${ }^{1}$, D.V. Leybo ${ }^{1}$, D.S. Pankratova ${ }^{1}$, A.T. Burkov' ${ }^{2}$, V.V. Khovaylo ${ }^{1}$

${ }^{1}$ National University of Science and Technology „MISIS“, 119049 Moscow, Russia

${ }^{2}$ loffe Institute, 194021 St. Petersburg, Russia

Abstract The work presents experimental results on the thermoelectric properties of the bulk $p$-type oxyselenides $\mathrm{Bi}_{1-x} \mathrm{Pr}_{x} \mathrm{CuSeO}$ $(x=0,0.04,0.08)$ and $\mathrm{Bi}_{0.96} \mathrm{La}_{0.04} \mathrm{CuSeO}$, fabricated by the solidstate reaction technique. The temperature dependences of the thermopower, the electrical resistivity, and the thermal conductivity were measured from room temperature to $800 \mathrm{~K}$. In the whole temperature range, decrease of the electrical resistivity, and the thermopower with increasing substitution level was observed, while the thermal conductivity was almost unaffected by the doping. Despite the fact that the nominal valence of $\mathrm{Bi}, \mathrm{La}$ and $\mathrm{Pr}$ is the same, the replacement of bismuth with rare-earth ions leads to increase of the charge carrier concentration, which may be caused by the difference in the electron configurations of ions, resulting in the Fermi level shift to the valence band. 